\title{
Equipamiento de laboratorio para mejorar el aprendizaje en comunicaciones móviles
}

\author{
Almudena Díaz Zayas, Pedro Merino Gómez \\ Departamento de Lenguajes y Ciencias de la Computación, \\ Universidad de Málaga, Andalucía Tech, \\ Edificio de Investigación Ada Byron, Málaga, España, 29071. \\ almudiaz@lcc.uma.es, pedro@lcc.uma.es
}

\author{
F. Javier Rivas Tocado \\ Keysight Technology \\ Málaga, Spain \\ Email: javi_rivas@keysight.com
}

\begin{abstract}
Resumen-Se espera que las generaciones venideras de graduados e investigadores de ingeniería desarrollen un conjunto integral de habilidades para adaptarse a la industria de las comunicaciones móviles y a las expectativas del mercado. Para satisfacer estas demandas las universidades tendrán que colaborar e innovar en sus estilos de educación, herramientas de investigación y procesos de aprendizaje. La evolución de la experimentación remota y la disponibilidad de sistemas avanzados de pruebas para comunicaciones móviles ofrecen nuevas oportunidades, como la experimentación con la clase Gigabit LTE-A. En este artículo discutimos cómo un emulador de LTE-A podría ser adoptado con fines educativos y cómo se pueden usar tecnologías de acceso a entornos de investigación federados para proporcionar un acceso remoto y controlado.
\end{abstract}

Palabras Clave-comunicaciones móviles, docencia, emulador de estación base, laboratorios remotos, experimentación

\section{INTRODUCCIÓN}

La rápida evolución y adopción de LTE y la nueva especificación 5G New Radio (NR) son indicativas de la velocidad con la que se avanza en el sector de las tecnologías móviles. Cada nuevo desarrollo tecnológico trae consigo nuevos desafíos que las nuevas generaciones de ingenieros tendrán que afrontar. Por ejemplo, los dispositivos móviles, las aplicaciones y los protocolos tienen que lidiar con velocidades de datos muy elevadas ( superior a $10 \mathrm{GHz}$ ) que pueden requerir nuevos diseños y arquitecturas, tanto hardware como software. En este sentido, los recursos educativos disponibles en las universidades deben permitir desarrollar estas nuevas competencia. En un campo en el que la tecnología evoluciona de una forma tan rápida es fundamental que los futuros ingenieros reciban una formación lo más actualizada posible y que los investigadores tengan acceso a instrumentos de vanguardia para obtener resultados relevantes.

En [1] Castro et al. se lleva a cabo un análisis de la evolución y avances que han tenido lugar en las técnicas educativas utilizadas en el ámbito de las titulaciones de ingeniería en la última década. En su estudio destacan que, en el pasado, las Universidades habían exhibido un cierto inmovilismo en atender las necesidades específicas de la industria. Sin embargo, se está produciendo un cambio de paradigma debido al uso de Internet y de las tecnologías móviles para proporcionar oportunidades de aprendizaje ubicuas y a lo largo de toda la vida profesional centradas en el estudiante.

La necesidad de conseguir una educación actualizada y orientada a las necesidades vigentes en la industria no es específica del dominio de las telecomunicaciones. TATU [2] es un ejemplo de un proyecto del programa TEMPUS de la Unión Europea que apoya la modernización de la educación superior en paises socios de la Unión Europea y pertenecientes a zonas de Europa Oriental, Asia Central, los Balcanes occidentales y la región mediterránea. El proyecto TATU tiene por objetivo mejorar la empleabilidad de los graduados universitarios a través del establecimiento, en las universidades, de centros de entrenamientos especializados equipados con modernos laboratorios de tecnologías punteras en el sector de la automoción.

Por otro lado, y ya en el contexto de las comunicaciones inalámbricas, el trabajo presentado en [3] es un ejemplo significativo de la integración de sistemas de gestión de aprendizaje tradicionales con el acceso remoto a instrumentación real ubicada en laboratorios de comunicaciones inalámbricas.

La experimentación remota a distancia está recibiendo cada vez más atención ya que maximiza el uso de los equipos, aumenta la disponibilidad de los laboratorios y permite mejorar el aprendizaje a distancia proporcionando entornos para la ejecución de prácticas [1][3][4][5][6]. Existen diferentes tipo de laboratorios remotos, muchos de ellos basados en experimentos simulados mediante entornos web virtuales. El uso de simuladores es útil para reducir costes, pero existen argumentos sobre su 


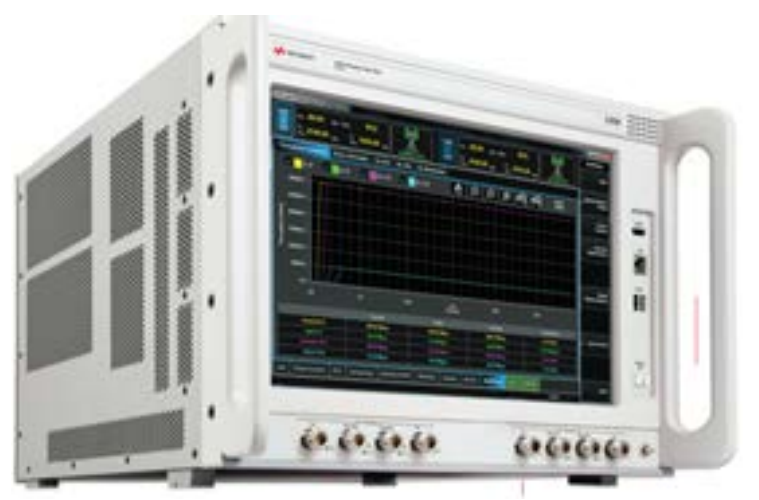

Fig. 1. UXM wireless test set

efectividad comparada con el acceso a equipamiento real [7], por lo que debería combinarse con laboratorios reales para conseguir entornos de aprendizaje para estudiantes más efectivos [4].

En ese sentido existen también laboratorios de experimentación remota que incluyen instrumentos reales como generadores de señal y analizadores de señal que permiten generar y medir señales de RF [8]. En [5] se resaltan los beneficios de los laboratorios que proporcionan acceso desde Internet para conseguir un eficiente uso de los equipos de radiocomunicación. Como parte de este trabajo los autores han realizado también una evaluación de los efectos positivos que tienen sobre el aprendizaje el uso de laboratorios remotos y los beneficios que tienen para los estudiantes en términos de flexibilidad y mayor disponibilidad.

Sin embargo, los autores del presente trabajo no tienen constancia de la existencia de ningún laboratorio de educación remota que proporcione acceso a emuladores de redes móviles que permiten configurar y analizar de cerca el funcionamiento real de los teléfonos móviles en un entorno realista y a la vez controlado.

El resto del artículo está organizado de la siguiente forma. La sección II proporciona una descripción detallada de la principales características proporcionadas por el UXM Wireless Test Set, y cómo éstas pueden ser utilizadas para mejorar el aprendizaje de los alumnos en asignaturas de comunicaciones móviles. En la sección III se muestra cómo la Universidad de Málaga ha integrado dicho sistema en el testbed PerformNetworks el cual puede ser accedido remotamente, a través de tecnologías y protocolos FIRE (Future Internet Research y Experimentación), tanto para experimentación como para educación.

\section{EMULACIÓN DE REDES MÓVILES}

En este artículo planteamos el uso de sistemas avanzados de pruebas para comunicaciones móviles como el UXM Wireless Test Set de Keysight Technologies, que se muestra en la Figura 1 en labores docentes y de investigación. Estos equipos son, tradicionalmente, usados por fabricantes de terminales móviles, operadores y laboratorios de certificación para ejecutar las pruebas de conformidad definidas por organismos de estandarización

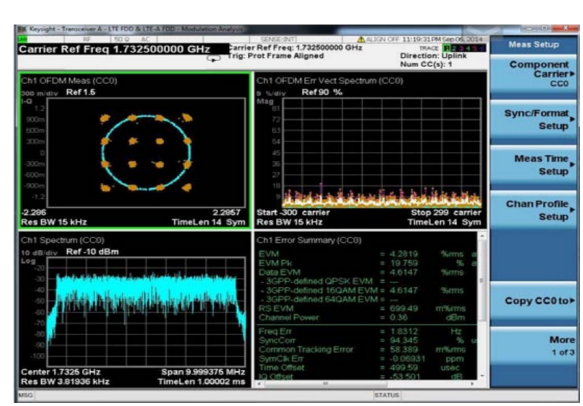

Fig. 2. Herramientas de medidas XApps

como el 3GPP (3rd Generation Partnership Project) y que deben pasar los terminales móviles antes de ser lanzados al mercado. Estos equipos se comportan como estaciones base reales desde el punto de vista de los teléfonos móviles que se están probando, además de proporcionar una gran variedad de funcionalidades destinadas a proporcionar un entorno de pruebas de I + D potente y flexible.

\section{A. Uso del UXM en actividades de laboratorio}

El UXM es un instrumento extremadamente versátil. Tiene la capacidad de emular varias estaciones base con diferentes tecnologías de acceso radio, incluyendo LTE/LTE-A, WCMS/HSDPA+, GSM/GPRS/EGPRS y TD-SCDMA/HSPA. También puede operar simultáneamente como un emulador de canal radio,ruido y generador de formas de onda arbitrarias para generar interferencias y degradación controlada y como un analizador de señal.

Una de las ventajas más destacadas del UXM es la facilidad de uso del E7530A y E7630A LTE/LTE-A Test and Lab Applications [9] que se ejecutan en el propio instrumento. Para el acceso en modo local el instrumento ofrece un interfaz de usuario basado en ventanas diseñado para ser usado desde la pantalla táctil del instrumento. También se puede acceder a la interfaz gráfica del instrumento remotamente usando el protocolo RDP (Remote Desktop Protocol). Para controlar remotamente el equipo también se puede recurrir a la interfaz SCPI (Standard Commands for Programable Instruments) que ofrece.

A continuación introduciremos brevemente las características claves del UXM y el potencial que éstas tienes para su uso en tareas educativas y de investigación en comunicaciones móviles. Cada característica podría ser la base para el diseño de diferentes laboratorios experimentales.

1) Análisis de transmisión de RF y banda base: Entre sus muchas capacidades, el UXM proporciona soporte nativo de las soluciones de medida Keysight, que son el estándar de-facto para análisis de señal. A través de ellas es posible introducir a los estudiantes a multitud de conceptos, como la medida de Magnitud del Vector Error (EVM), análisis de espectro, ecualización y planicidad del canal, análisis de modulación IQ y muchos más. La Figura 2 muestra una medida de análisis de modulación de una señal de $20 \mathrm{MHz}$ con modulación 16QAM en el enlace 


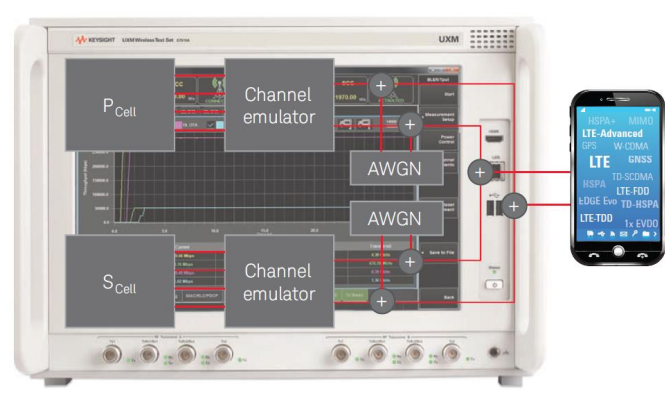

Fig. 3. Emulation de canal digital

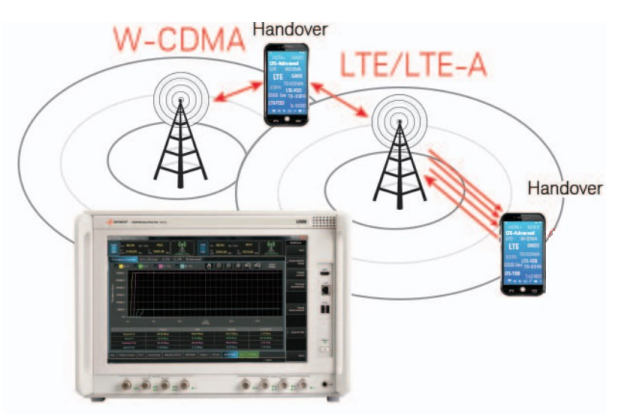

Fig. 4. Procedimientos de movilidad

ascendente.

2) Emulación de canal y Análisis de rendimiento de recepción: Los receptores de los dispositivos móviles se evalúan típicamente en términos de la probabilidad de recepción de datos en presencia de interferencia y degradación. El porcentaje de error resultante se chequea contra umbrales de evaluación definidos.

Para replicar el efecto de las condiciones de propagación radio, el UXM incorpora un emulador de canal digital. Esta funcionalidad proporciona gran facilidad de uso y mejora la precisión evitando las contribuciones de incertidumbre típicamente asociadas a un número elevado de interconexiones RF.

Además de la emulación de escenarios de propagación multicamino, se pueden generar degradaciones adicionales de la transmisión incluyendo generación de señales de continua $(\mathrm{CW})$, ruido blanco gaussiano (AWGN) y formas de onda arbitrarias.

La Figura 3 muestra un diagrama lógico de la emulación de canal y la generación de interferentes para una configuración de dos celdas portadoras (CC) con una configuración de antenas MIMO 4×2. Representando 4 antenas de transmisión en la estación base y 2 antenas de recepción en el terminal móvil. Otros escenarios posibles en un mismo UXM incluyen hasta $4 \mathrm{CC}$ con $2 \mathrm{x} 2 \mathrm{MIMO}$ o $2 \mathrm{CC}$ con $4 \mathrm{x} 4 \mathrm{MIMO}$.

3) Procedimientos de movilidad: Como se muestra en la Figura 4, es posible generar múltiples celdas en un mismo UXM, incluyendo diferentes tecnologías de acceso radio (RAT), proporcionando un interfaz táctil intuitivo y fácil de usar.

Entender los entresijos de los protocolos de señalización de las redes móviles, puede ser una tarea compleja si no se se cuenta con ejemplos reales de funcionamiento. El uso de un equipo como el UXM puede ser la forma ideal de mejorar la curva de aprendizaje en este tipo de escenarios.

Además de introducir conceptos básicos como la planificación del espectro radio, las bandas de frecuencia y los distintos anchos de canal, es extremadamente sencillo realizar y supervisar procedimientos de movilidad como el registro de red, el deregistro y la reselección de celda. Otros escenarios más avanzados de interés pueden ser los diferentes tipos de traspasos en la misma o distintas bandas de frecuencia, o incluso entre distintas tecnologías radio. Como se ha comentado anteriormente, es posible analizar el impacto del canal radio y de las interferencias de forma realista pero manteniendo control de las mismas.

4) Análisis de rendimiento de comunicaciones IP extremo a extremo: Los teléfonos inteligentes o smartphones hace tiempo que dejaron de usarse únicamente para realizar llamadas de voz. En su lugar predominan actualmente las comunicaciones multimedia basadas en el protocolo IP. Otra característica clave de los usos actuales es la amplia variedad de perfiles de usuarios y de patrones de tráfico.

En este escenario, es importante asegurar que los dispositivos móviles puedan transmitir datos a su máxima capacidad. La capacidad del UXM evoluciona continuamente para ayudar a investigadores e ingenieros a entender las interacciones entre aplicaciones, sistemas operativos y las pilas de protocolos.

Asímismo, es posible entender en profundidad las dinámicas de tráfico E2E mediante el uso de gráficas de monitorización de tiempo real. Este tipo de representaciones permite observar de forma simultánea la evolución tanto de la velocidad de las transmisiones IP como de las tasas de transmisión de capa MAC. La Figura 5 contiene gráficas de transmisión para un escenario de $3 \mathrm{CC}$ con 2x2 MIMO y una modulación 256QAM en el enlace descendente. Como resultado, los 6 flujos MIMO resultantes pueden transportar cerca de $600 \mathrm{Mbps}$.

Este tipo de representación resulta particularmente útil para identificar artefactos de tráfico que pueden relacionar interacciones entre capas que afectan al rendimiento de las comunicaciones a nivel de aplicación.

5) Monitorización y análisis de protocolos: El UXM incorpora una potente herramienta software de monitorización y análisis [10], la E7515A-L01. Este software permite a los usuarios del UXM controlar la generación y filtrado de trazas a través de un interfaz integrado con wireshark.

Además de analizar los protocolos de señalización de capas altas, como los de gestión de recursos radio (RRC) y del núcleo de red (NAS), es posible acceder a la información de control y a los bloques de datos (PDU) del protocolo de acceso al medio (MAC).

La Figura 6 proporciona un ejemplo de intercambio de mensajes RRC, como los que se usan para difundir la información de celda y para establecer las conexiones radio, así como información detallada sobre la operativa 


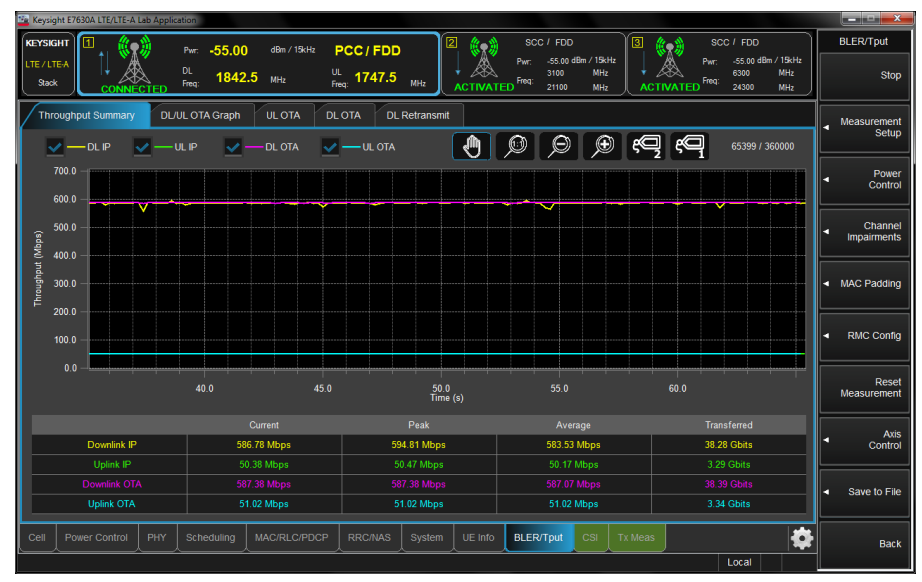

Fig. 5. E2E IP and MAC level throughput graphs

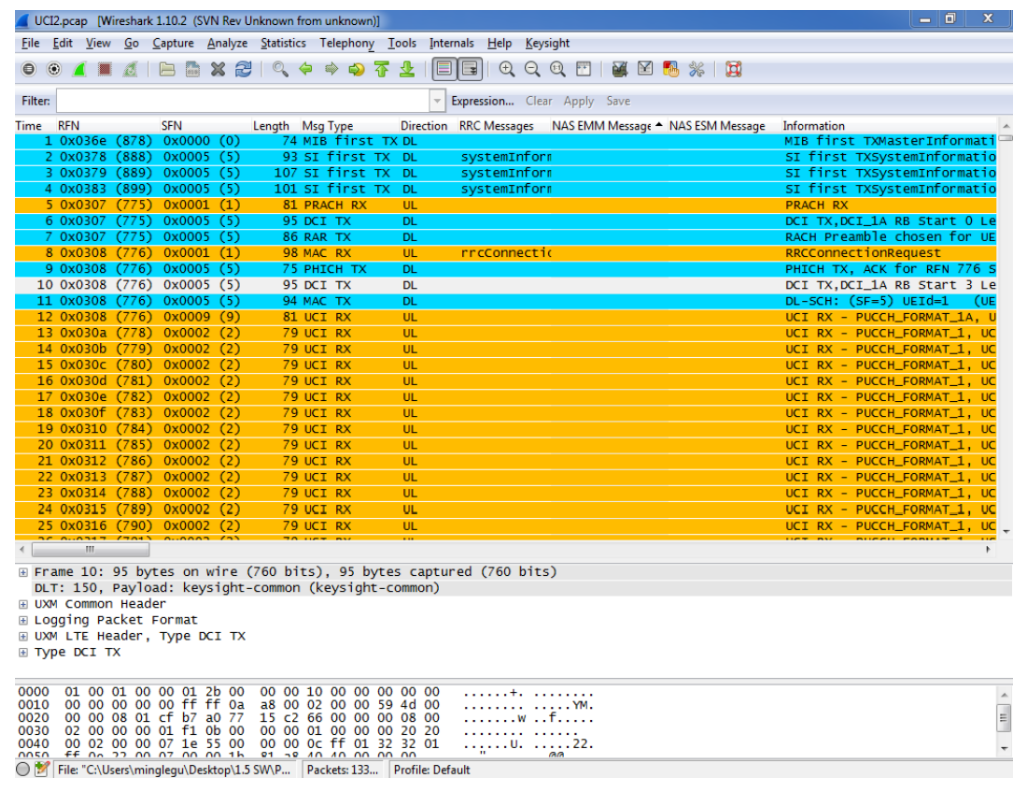

Fig. 6. Software de logging y análisis de protocolos

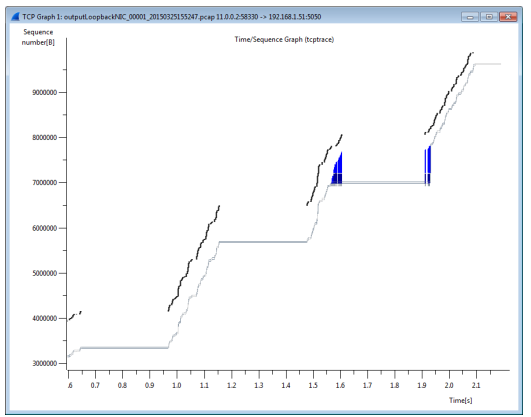

Fig. 8. Integración con herramientas de Wireshark para el análisis de flujos de datos

de los canales de control.

Se pueden analizar escenarios de tráfico complejos, y entender los mecanismos de detección de error y retransmisión de las distintas capas de comunicación, forzando la aparición de errores de comunicación mediante interferentes y canales variantes.

Resulta particularmente interesante el uso de las capacidades integradas de análisis de flujos para estudiar la dinámica de las conexiones TCP a partir de los mensajes MAC_LTE, como se muestra en la Figura 7.

6) VoLTE y Multimedia: El UXM proporciona múltiples funciones de acceso radio que son necesarias para la transmisión de voz sobre LTE mediante el perfil VoLTE [11], incluyendo la planificación semi-persistente (SPS), el uso de múltiples portadoras radio (DRBs), la recepción discontinua (DRX) y otras más.

LTE se basa en una red IP extremo a extremo y hace uso del Subsistema Multimedia IP (IMS) para mantener un registro de terminales (UEs) compatibles con IMS y para establecer sesiones multimedia.

El UXM integra un servidor IMS-SIP [12] que puede combinarse con un cliente software IMCS-SIP para probar escenarios VoLTE y otros servicios multimedia. La Figura 


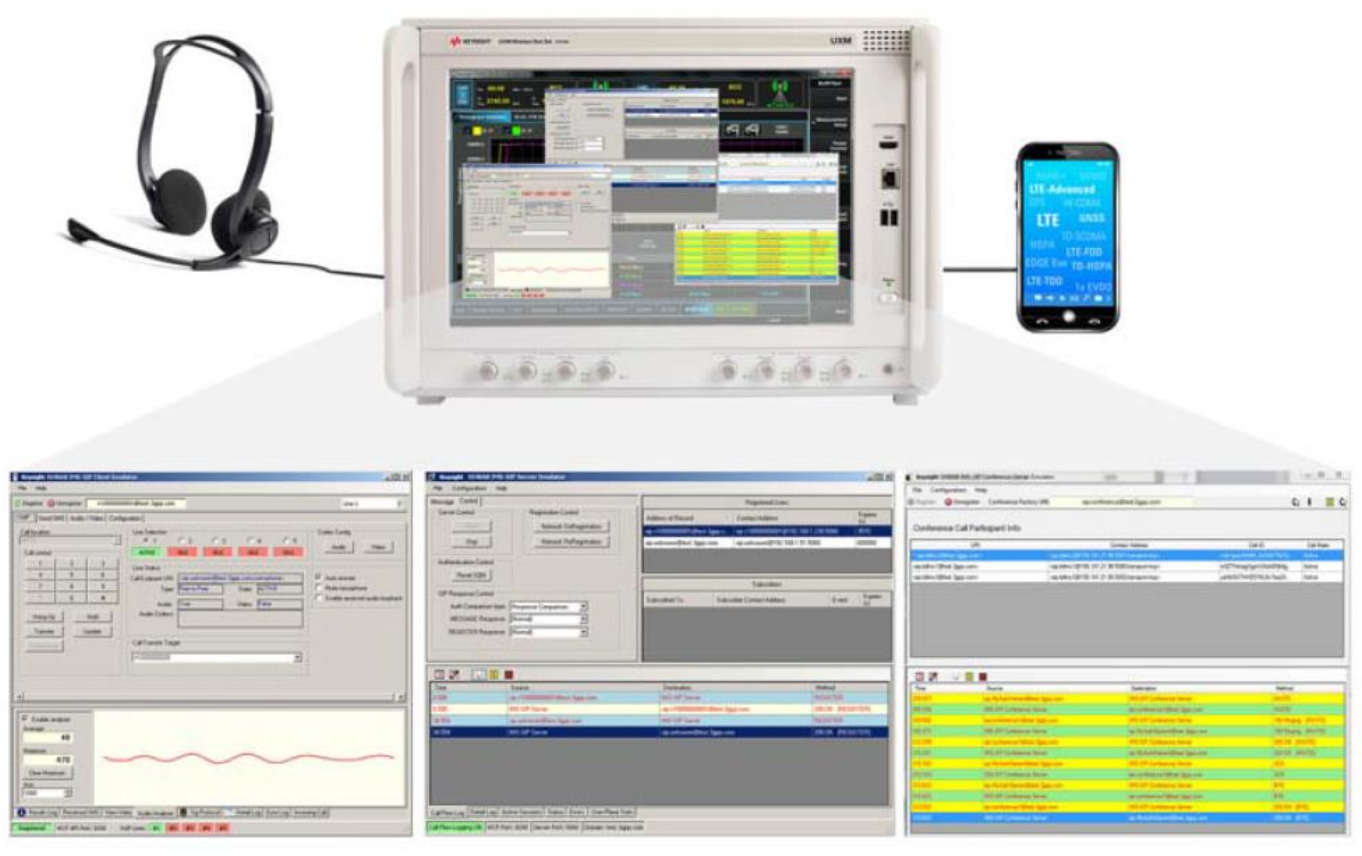

Fig. 7. Herramientas IMS-SIP y VoLTE

(F)elCIC aggressor test

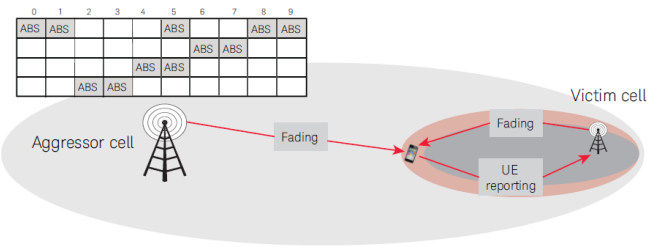

Fig. 9. Redes heterogéneas con eICIC

8 ilustra las diferentes herramientas para prueba de audio sobre comunicaciones IP.

7) Redes heterogéneas: Las redes heterogéneas han sido un campo de investigación con gran actividad en los últimos años. Estos escenarios combinan típicamente múltiples celdas con diferentes potencias, de forma que las distintas celdas se coordinan entre sí. De esta forma, en distintos instantes temporales, unas celdas transmiten mientras que las otras reducen al mínimo la interferencia que generan. Así se consigue mejorar la calidad de las conexiones especialmente en las fronteras entre celdas. Este tipo de técnicas se conoce como Coordinación de Interferencia Entre Celdas Mejorada (eICIC).

Para este fin, la red configura a los dispositivos móviles para que midan la potencia y calidad de señal de distintas celdas en diferentes instantes de tiempo. Se usa un patrón de transmisión especial de baja potencia, también llamado de subtramas casi vacías (ABS), para reducir la interferencia causada a las celdas vecinas tanto en la recepción de datos como en la estimación de canal. Con un correcto alineamiento de los períodos de medida a los patrones de transmisión $\mathrm{ABS}$, los terminales pueden solicitar dos esquemas de modulación y codificación (MCS) diferentes para que la red se ajuste a los distintos niveles de interferencia. Así pues, en las subtramas en las que las celdas vecinas reducen su interferencia, la calidad del canal observado será mejor y será posible utilizar una modulación de mayor capacidad de transmisión.

La Figura 9 muestra un ejemplo de un patrón de interferencia de $40 \mathrm{~ms}$, donde una celda agresora intercala períodos de transmisión a potencia completa con subtramas ABS. La transmisión completa causa una mayor interferencia desde la agresora, mientras que las subtramas ABS permiten al teléfono recibir la señal de la celda víctima con una menor interferencia.

Las especificaciones de prueba del 3GPP definen un conjunto de patrones de referencia y de configuraciones de celda, pero el UXM proporciona un interfaz altamente flexible para explorar el rendimiento de escenarios de 


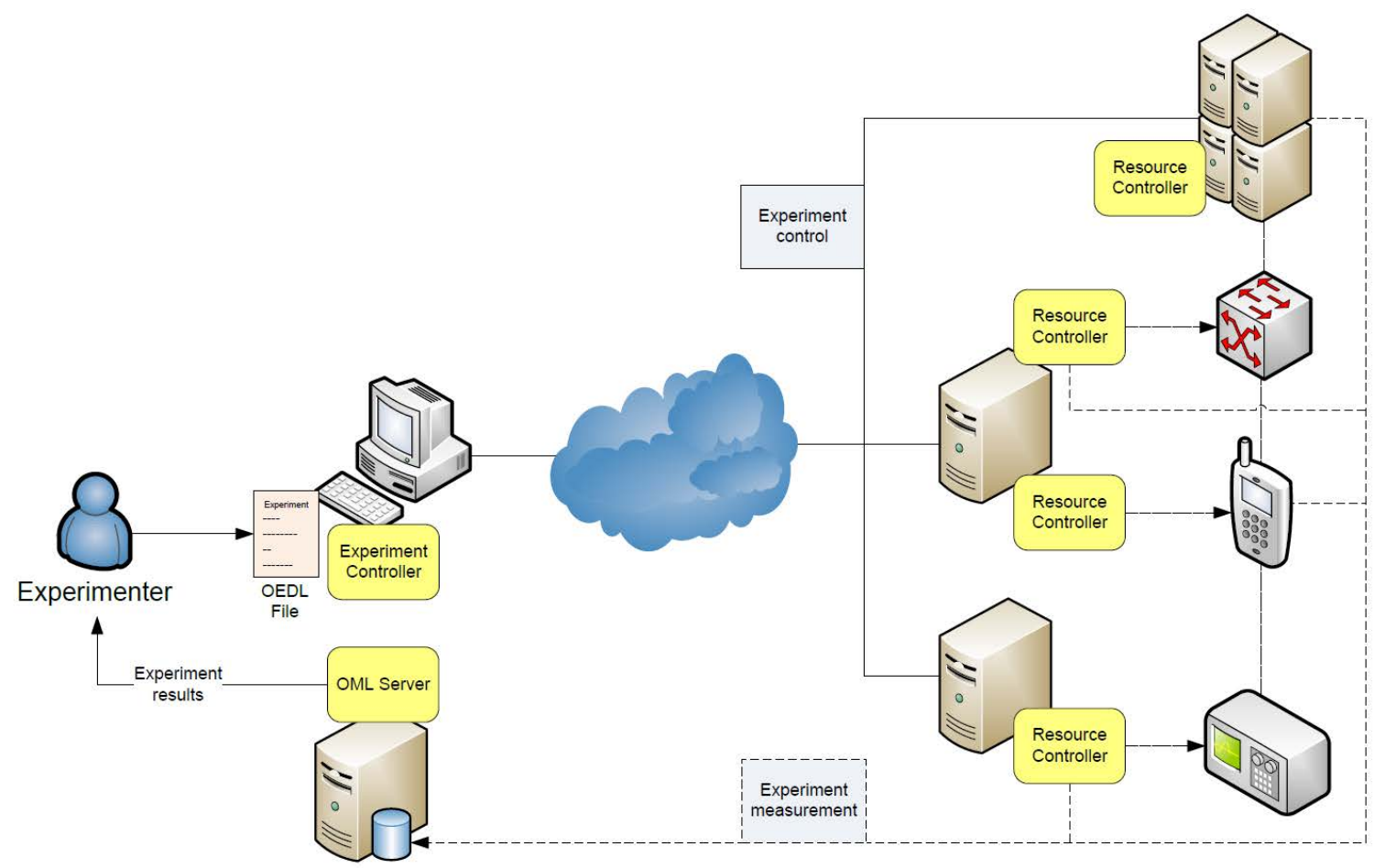

Fig. 10. Arquitectura de un testbed GENI/FIRE

agresión complejos. Es posible definir distintos escenarios ABS para reducir la potencia, donde típicamente se transmite al menos las señales de referencia específicas de la celda (CRS) para que los móviles acampados en la celda puedan funcionar.

\section{PERFORMNETWORKS, UN TESTBED DE EXPERIMENTACIÓN REMOTA}

En esta sección se introduce el testbed PerformNetworks [13] del grupo MORSE de la Universidad de Málaga. Dicho testbed está enfocado en la experimentación entorno a las comunicaciones móviles. PerformNetworks proporciona un entorno real y controlado compuesto por el emulador de estación base introducido en la sección anterior, teléfonos móviles comerciales, analizadores de potencia, un núcleo de red LTE y small cells, como elementos más destacados. Se puede consultar la descripción detallada y actualizada del testbed en http://morse.uma.es/performnetworks.

En esta sección se ilustra cómo se ha usado la tecnología FIRE para dar acceso remoto al testbed. La adopción de esta tecnología ha permitido que incorporar el testbed en la infraestructura de investigación europea promovida por el proyecto europeo Fed4Fire. También se explica el flujo de trabajo de la orquestación de un experimento.

\section{A. Tecnologías para el control de recursos de laboratorio}

GENI y FIRE son dos iniciativas que tratan de crear un entorno de experimentación común, la primera en Estados Unidos y la segunda en Europa. Ambas iniciativas se basan en la misma idea: la evaluación experimental es un requisito indispensable para proporcionar resultados significativos que puedan ser aplicados en el mundo real. Así, el concepto de testbed experimentales es fundamental tanto en la comunidad GENI como en la comunidad FIRE. Cabe destaca que aunque GENI y FIRE surgieron como dos iniciativa independientes, el proyecto Fed4Fire ha adoptado las tecnologías GENI, haciéndolas compatible con las tecnologías FIRE.

La investigación experimental requiere la capacidad de realizar experimentos de una manera fácil y repetible. En el testbed PerformNetworks se ha adoptado OMF y OML como soluciones para proporcionar control y medición de experimentos, respectivamente. La arquitectura de un testbed genérico basado en estas tecnología se muestra en la Figura 10. Un experimento se define como un archivo escrito en el Lenguaje de descripción de experimentos de OMF (OEDL). Un archivo OEDL declara los recursos que utilizará el experimento, los eventos a los que reaccionará y las acciones a realizar en respuesta a dichos eventos.

El controlador del experimento (EC) interpreta los scripts OEDL y coordina la ejecución del experimento. Cada recurso del testbed es administrado por un controlador de recursos (RC), que puede alojarse en un ordenador externo o en el propio recurso. Los RCs y los ECs intercambian información de control utilizando el Protocolo de Control de Recurso Federado (FRCP), que puede ser transportado a través del protocolo XMPP (Extensible Messaging and Presence Protoco) o del protocolo AMQP (Advanced Message Queuing Protocol).

El servidor OML recopila y almacena las medidas del experimento. Cada instrumento, o un RC en nombre de un instrumento, puede enviar las medidas a través de un cliente OML. Para la implementación de dicho cliente 


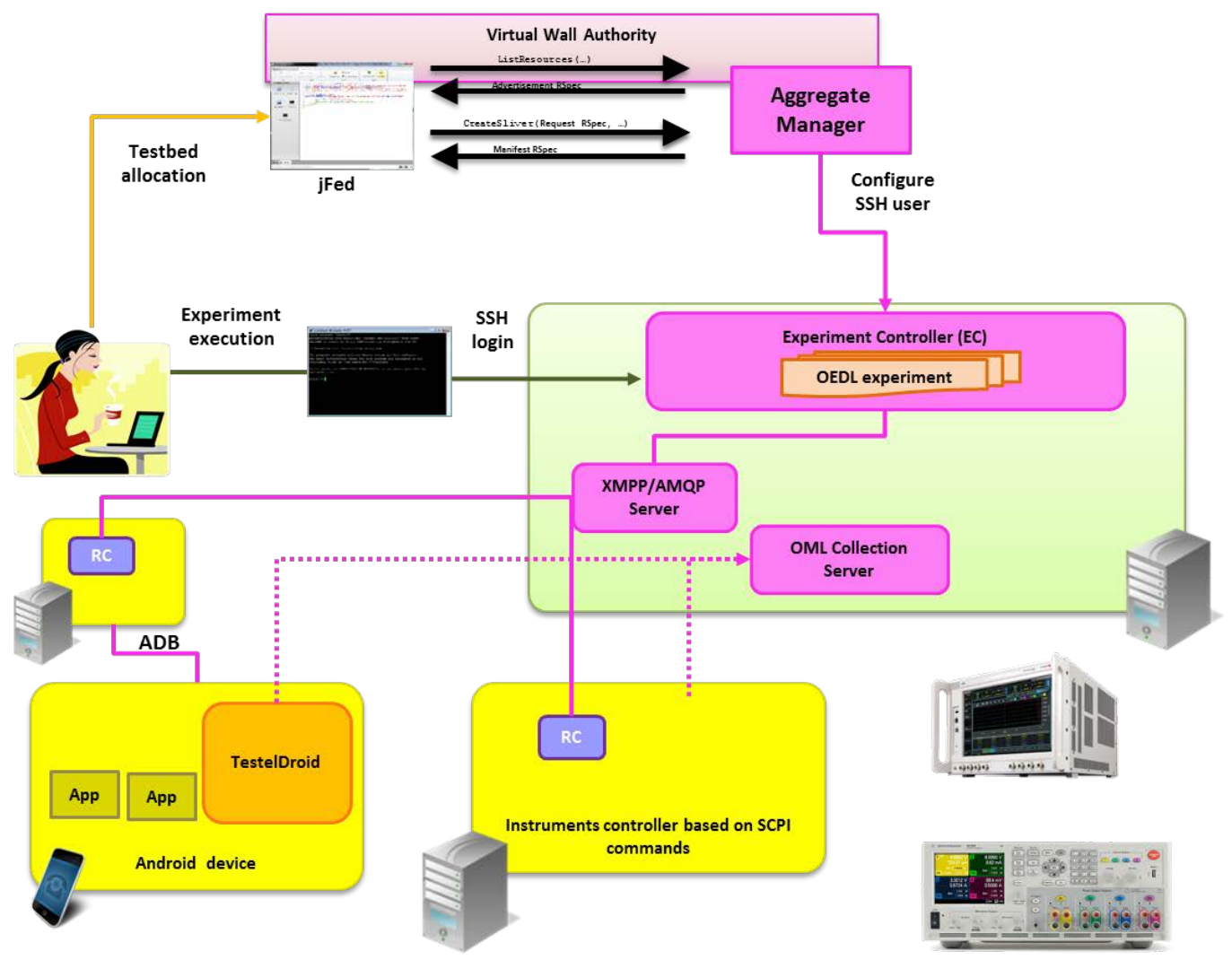

Fig. 11. Acceso remoto y control de la experimentación en el testbed PerformNetworks

Código 1. Ejemplo de script OEDL

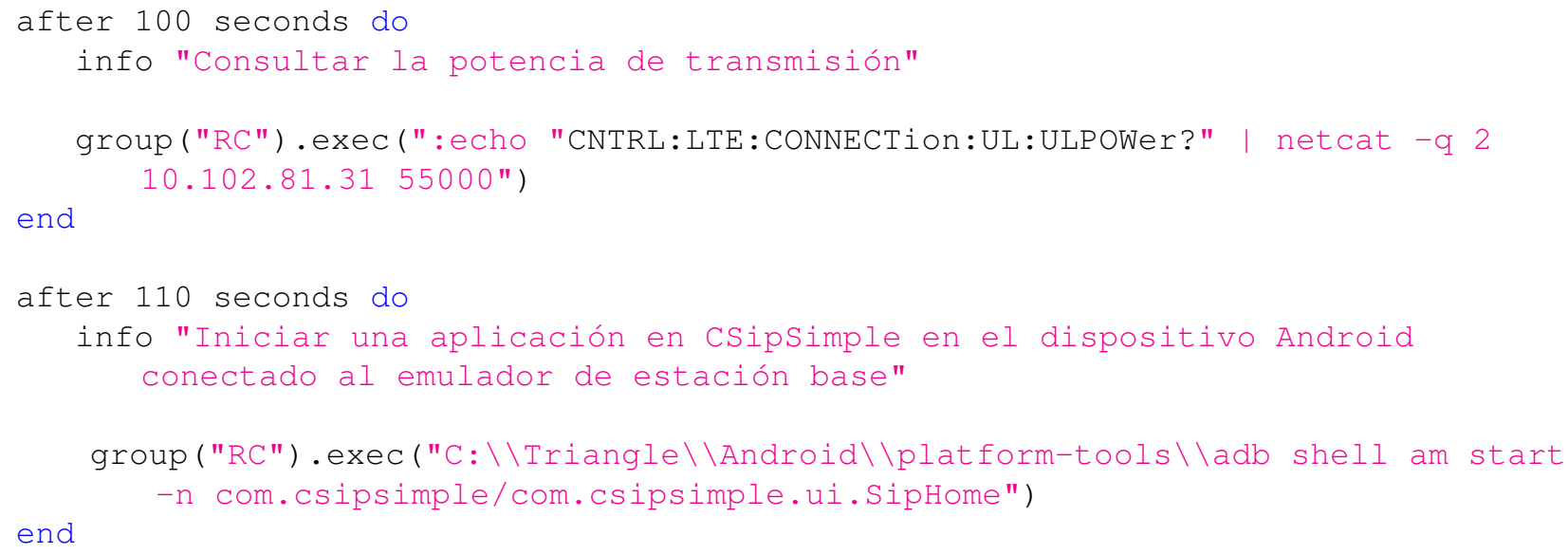

se proporcionan librerías en distintos lenguajes: C, Ruby, Java, etc. El servidor OML tiene como backend una base de datos PostGreSQL de forma que las medidas almacenadas pueden ser consultadas utilizando herramientas SQL estándar.

Una vez que se ha definido el experimento, se envía al EC. El EC interactúa con los RCs de los recursos implicados en el experimento, por ejemplo un móvil Android o el emulador de estación base, para ejecutar en ellos las acciones definidas en el script. En el script OEDL también se definen los puntos de medidas del experimento, las medidas serán almacenadas en el servidor OML.

El descubrimiento, la reserva y el aprovisionamiento de los recursos disponibles en el testbed se realiza a través de SFA (Slice-based Facility Architecture) [14]. Para iniciar los procesos de descubrimiento y reserva necesitamos proporcionar un especificación de nuestro recurso denominada RSpec. La entidad definida por el SFA para gestionar las consultas sobre los recursos es el Aggregate Manager (AM). El AM es consultado por una herramienta cliente que implementa el API de comunicación con el AM, en nuestro caso usamos jFed [15]. 


\section{B. Flujo de trabajo de experimentos ejecutados remota- mente en PerformNetworks}

El flujo de trabajo para acceder remotamente al testbed se muestra en la Figura 11. El primer paso es obtener un certificado X.509 a través de la autoridad de autenticación de Fed4Fire, accesible en https://authority.ilabt.imind.be. Este certificado es proporcionado a la herramienta jFed para que pueda establecer una comunicación con el AM y reservar un intervalo temporal para acceder al testbed. Una vez realizada la reserva del testbed el AM de PerformNetworks proporciona un acceso SSH federado al EC que gestiona los recursos del testbed.

Se proporcionan scripts de referencia que muestran cómo controlar el UXM y las aplicaciones que se ejecutan en el móvil (ver extracto de script que se muestra en Código 1). En los scripts se usan los procedimientos disponibles en el RC asociado a cada recurso del testbed para enviarle, en el caso de los instrumentos, los comandos SCPI definidos en el manual de usuario, o , en el caso de los dispositivos móviles Android, comandos ADB, que permiten configurar el móvil y lanzar aplicaciones.

Una vez editados los experimentos pueden ser ejecutados usando el procedimiento omf_ec (ver Código (2)) disponible en el EC al cual se tiene acceso mediante SSH.

\section{CONCLUSIONES}

Para mantener el ritmo al que las tecnologías móviles evolucionan se necesitan nuevas herramientas y nuevos enfoques en las Universidades y en los centros de investigación. La utilización de equipos como el UXM Wireless Test Set permitirán a los estudiantes desarrollar y mantener sus competencias actualizadas.

En el presente artículo se ha ilustrado la forma de integrar este equipo en un testbed más amplio con acceso remoto. También se ha demostrado su uso remoto a través de los protocolos de experimentación promovidos por la comunidad de testbed europea FIRE.

\section{AGRADECIMIENTOS}

Este trabajo ha sido financiado por el Ministerio Español de Economía y Competitividad (TIN2015-67083R), FEDER y el programa de investigación e innovación Horizonte 2020 de la Unión Europea (grant agreement No 688719).

\section{REFERENCIAS}

[1] Castro M.; Tawfik M.; Tovar E., "Digital and Global View of Engineering Education Using Remote Practical Competences", IEEE Revista Iberoamericana de Tecnologias del Aprendizaje (RITA) (Volume:10 , Issue: 3 ) pp 126-133. doi: 10.1109/RITA.2015.2452651

[2] Workshop: "The TATU Lab \& Smart Education", 2016 13th International Conference on Remote Engineering and Virtual Instrumentation (REV), pp 400-402
[3] Kafadarova N.; Mileva N.; Stoyanova S., "Remote Wireless Communications lab in real time". 2013 IEEE Global Engineering Education Conference (EDUCON), pp 69-74

[4] Tawfik M.; Sancristobal E.; Martin S.; Gil R.; Pesquera A.; Albert M.J.; Peire J.; Milev M.; Mileva N.; OSuilleabhain G.; Tzanova S.; Kreiner C.; Hormann L.B; Castro M., "Labor-Oriented Online Master Degree Program", 2013 IEEE Global Engineering Education Conference (EDUCON), pp 1098-1102

[5] Gampe, A; Melkonyan, A; Pontual, M;Akiopian D.; "An Assessment of Remote Laboratory Experiments in Radio Communications", 2014, IEEE Transactions on Education, Volume:57, Issue: 1 , pp $12-19$

[6] Nassar, A.; Mohammed, M.; Elrashidi, A.; Elleithy, K.; "Virtual Wireless and Mobile Communication Laboratory, Education", Vol. 2 No. 1, 2012, pp. 19-24. doi: 10.5923/j.edu.20120201.04.

[7] Feisel, L. D.; and Rosa, A.J.; "The role of the laboratory in undergraduate engineering education”, Journal Eng. Edu., vol. 94, no. 1, pp. 121-130

[8] Kara, A.; Aydin, E.U.; Oktem, R.; Cagiltay, N., "A Remote Laboratory for Training in Radio Communications: ERRL," in Personal, Indoor and Mobile Radio Communications, 2007. PIMRC 2007. IEEE 18th International Symposium on , vol., no., pp.1-5, 3-7 Sept

[9] Keysight Technologies. E7515A UXM Wireless Test Set [Online]. Available: www.keysight.com/find/uxm, visited on 1 January 2016.

[10] Keysight Technologies, E7515A-L01 Protocol logging and analysis software, User's guide. 2014

[11] Official Document IR.92 - IMS Profile for Voice and SMS. GSM Association. 2015.

[12] Keysight Technologies,E6966B IMS-SIP network emulator, Technical Overview. 2015

[13] Díaz Zayas, A.;Recio Perez A.M.; García Perez, C.A.; Merino P, PerformLTE: a Testbed for LTE testing in the Future Internet, 13th International Conference on Wired \& Wireless Internet Communications. 2015

[14] Slice-Based Federation Architecture, Version 2.0, GENI Initiative, Tech. Rep., Jul. 2010. [Online]. Available: http://groups.geni.net/geni/ wiki/SliceFedArch

[15] B. Vermeulen, W. Van de Meerssche, T. Walcarius, jFed toolkit, Fed4FIRE, Federation; GENI Engineering Conference (GEC) , 2014 